\title{
The diagnostic value of apparent diffusion coefficient to differentiate benign and malignant meningiomas
}

\author{
Risa Marissa $^{1^{*}}$, Rachmi Fauziah Rahayu ${ }^{2}$, Hari Wujoso ${ }^{3}$, Subandi $^{4}$, \\ Prasetyo Sarwono Putro ${ }^{2}$, and Widiastuti Soewondo ${ }^{2}$
}

\begin{abstract}
BACKGROUND
Meningiomas are the most common primary extra-axial non-glial intracranial tumors. The severe grade of meningioma, according to WHO, has the highest recurrence rate accompanied by high morbidity and mortality rates. Therefore, it is imperative to perform pre-operative assessments so the clinician can give prompt treatment to gain a better prognosis. It is a novel alternative way of predicting meningioma's malignancy by calculating the tumor's apparent diffusion coefficient (ADC) value. The objective of the study was to determine the value of ADC for differentiating benign and malignant meningiomas.
\end{abstract}

\section{METHODS}

This cross-sectional study involved 32 subjects with clinically diagnosed or histologically verified meningioma ( 21 benign and 11 malignant). They underwent a head-magnetic resonance imaging (MRI) examination and biopsy. We calculated the ADC value by creating regions of interest (ROIs) on the solid part of the tumor, guided by contrast and fluid-attenuated inversion recovery (FLAIR) sequence. We analyzed the ADC value with independent t-test and Bland-Altman graphs, calculated the average difference, CI 95\%, limit of agreement between observers, and ROC.

\section{RESULTS}

Mean ADC of malignant meningiomas $\left(0.877 \pm 0.167 \times 10^{-3} \mathrm{~mm}^{2} / \mathrm{s}\right)$ was significantly lower than that of benign meningiomas $\left(0.990 \pm 0.105 \times 10^{-3}\right.$ $\left.\mathrm{mm}^{2} / \mathrm{s}\right)(\mathrm{p}<0.05)$. The ADC threshold is $0.886 \times 10^{-3} \mathrm{~mm}^{2} / \mathrm{s}$ with sensitivity $63.6 \%$, specificity $85.7 \%$, positive predictive value $70 \%$ and negative predictive value $81.8 \%$.

\section{CONCLUSION}

The ADC value measurement provides a discriminative feature to differentiate between benign and malignant meningiomas. However, the clinical applicability still needs to be elucidated, as histopathological confirmation remains the mainstay of definitive diagnosis.

Keywords: Meningioma, apparent diffusion coefficient, magnetic resonance imaging, histopathological grade

${ }^{1}$ Resident of Radiology,

Faculty of Medicine,

Sebelas Maret University, Surakarta

${ }^{2}$ Radiology Department,

Faculty of Medicine, Sebelas Maret

University and dr. Moewardi

Hospital, Surakarta

${ }^{3}$ Forensic Department,

Faculty of Medicine, Sebelas Maret

University and dr. Moewardi

Hospital, Surakarta

${ }^{4}$ Neurology Department,

Faculty of Medicine, Sebelas Maret

University and dr. Moewardi

Hospital, Surakarta

*Correspondence:

dr. R. Risa Marissa

Jl. Sancang Raya No. 14, Babakan, Bogor Tengah, Bogor, West Java,

Indonesia, 16128

(+62) 811-135-985

Email:rmarissa@gmail.com

ORCID ID: 0000-0002-9306-7399

Date of first submission, January 24, 2021

Date of final revised submission, July

23, 2021

Date of acceptance, July 26, 2021

This open access article is distributed under a Creative Commons AttributionNon Commercial-Share Alike 4.0

International License

Cite this article as: Marissa R, Rahayu RF, Wujoso H, Subandi, Putro PS, Soewondo W. The diagnostic value of apparent diffusion coefficient to differentiate benign and malignant meningiomas. Univ Med 2021;40:138-47. doi: 10.18051/ UnivMed.2021.v40.141-150.

DOI: http://dx.doi.org/10.18051/UnivMed.2021.v40.141-150 Copyright@Author(s) - 


\section{INTRODUCTION}

Meningiomas are the most common intracranial non-axial primary tumors, ranging from 14 to $20 \%$ of all intracranial tumors and are common in middle-aged patients, with a male: female ratio of $1: 2$. $^{(1)}$

The incidence of meningiomas is $6: 100,000$ population, of which $2 \%-3 \%$ are asymptomatic, and $8 \%$ comprise multiple meningiomas. Most meningiomas are benign, slow-growing, and in some cases can be cured by surgery. Nevertheless, $10-20 \%$ show aggressiveness both clinically and histopathologically and cause symptoms that worsen with time. . $^{(2,3)}$

The World Health Organization (WHO) has divided meningiomas into three groups based on the histopathological findings. These groups are WHO grade I (typical) that occur in almost $90 \%$ of the population, WHO grade II (atypical) that have an incidence of $5 \%-7 \%$ in the population, and WHO grade III (anaplastic) that account for $1 \%-3 \%$ of the population. It is essential to assess the clinical prognosis to help clinicians determine the next treatment. For example, management of treatment for WHO grade I meningiomas can be observation or surgery. In contrast, the treatment for meningiomas of WHO grade II is total surgery with/without radiotherapy, and for meningiomas of WHO grade III is in the form of radical surgery accompanied by radiotherapy. The severest grade of meningioma, according to $\mathrm{WHO}$, has the highest recurrence rate accompanied by high morbidity and mortality rates and low life expectancy in the next five years. ${ }^{(2)}$ So, it is imperative to preoperatively assess the potential for invasive meningioma to determine appropriate treatment and improve the patient's prognosis. $^{(3)}$

Magnetic resonance imaging (MRI) can be the imaging technique of choice to picture intracranial meningiomas and determine the next treatment. Various ways have been taken to assess the grade of meningioma with MRI examinations at varying stages before surgery. ${ }^{(2)}$
However, conventional MRI cannot distinguish between benign and malignant meningiomas. The picture of heterogeneous lesions and contrast enhancement, margins indistinguishable from surrounding tissue, irregular edges, destruction of the surrounding bone, accompanied by peritumoral edema, leads to an image of atypical/malignant meningioma. Still, this is not specific because the picture can also be found in benign meningiomas. ${ }^{(4,5)}$

Diffusion-weighted imaging (DWI) in MRI examination is a non-invasive technique that can assess free fluid diffusion in tissues. The apparent diffusion coefficient (ADC) is a measure of the diffusion of water molecules in tissues and is calculated after determination of DWI in MRI. The ADC values are automatically computed by the software, resulting in a parametric map showing the rate of diffusion of water molecules, which will vary in each tissue. Measurements are made by drawing regions of interest (ROIs) on the ADC map. The ADC value is expressed in $\mathrm{mm}^{2} / \mathrm{s}$. Although there is no clear limit for the normal value, ADC values of under 1.0 to $1.1 \times 10^{-3} \mathrm{~mm}^{2} / \mathrm{s}$ are generally stated to be restricted. ${ }^{(6)}$ Moraru and Dimitrievici (7) state that the ADC value in cortical gray matter is $0.56-0.78 \times 10^{-3} \mathrm{~mm}^{2} / \mathrm{s}$, in white matter $0.319-0.686 \times 10^{-3} \mathrm{~mm}^{2} / \mathrm{s}$ and in cerebrospinal fluid $1.59-2.43 \times 10^{-3} \mathrm{~mm}^{2} / \mathrm{s}$.

Previous researchers have recognized the ADC value obtained from DWI as an effective method for distinguishing benign and malignant meningiomas. However, ADC values cannot differentiate between atypical and anaplastic/ malignant meningiomas. Fluid diffusion in body tissues depends on the ratio of extracellular to intracellular space. Atypical and malignant meningiomas have a high cell proliferation rate and cause reduced extracellular space, resulting in fluid diffusion restriction, ${ }^{(2,3)}$ which shows an increased signal intensity in DWI and decreased signal intensity in ADC. In previous studies conducted by Surov et al. ${ }^{(8)}$ and Azeemuddin et al., ${ }^{(9)}$ the ADC value played an essential role in determining the type of meningioma. 
For meningiomas with a soft consistency, management to remove the tumor is carried out using a suction probe. In contrast, meningioma with a hard consistency cannot be treated by tumor excision. The ADC boundary value between WHO grade I and II/III meningiomas varied in all previous studies. For example, the study conducted by Surov et al. ${ }^{(8)}$ used the value of $0.85 \times 10^{-3} \mathrm{~mm}^{2} / \mathrm{s}$ as the threshold to differentiate WHO grade I meningiomas, which have low proliferation rates, from the high proliferation potential contained in $\mathrm{WHO}$ grades II and III. The ADC assessment itself is still rarely done in the MRI examination protocol in our hospital, especially in meningioma patients. In some studies, as in the study conducted by Yiping et al., ${ }^{(10)}$ the ADC value can distinguish between benign and malignant meningiomas. However, there are several different results from previous studies, one of which is the study of Rad et al., ${ }^{(4)}$ where it said that benign and malignant meningiomas are indistinguishable by $\mathrm{ADC}$ values. We questioned the difference between ADC values in benign and malignant meningiomas because of the previous study results. The present study aimed to determine the value of ADC for differentiating benign and malignant meningiomas.

\section{METHODS}

\section{Research design}

This study was a cross-sectional study conducted in the Radiology Installation of $\mathrm{dr}$. Moewardi Hospital, Surakarta, from February 2020 to July 2020.

\section{Study subjects}

The target population consisted of meningioma patients who had undergone headMRI and histopathology examinations and were archived at dr. Moewardi Hospital, Surakarta, from March 2016 until April 2020.

\section{Sample size determination}

A total of 32 patients with clinically diagnosed or histologically verified meningiomas
( 21 benign and 11 malignant) were included. All samples were taken using purposive sampling techniques. Meanwhile, according to Memon et al., ${ }^{(11)}$ the appropriate sample size in a study is between 30 and 500. Even though the sample size between 30 and 500 at $5 \%$ confidence level is generally sufficient for many researchers, the decision on the size should reflect the quality of the sample in this wide interval. Therefore, the minimum number of samples set in this study was 30 samples. We only included patients that have undergone MRI and histopathology examination from March 2016 until April 2020. We excluded patients with en-plaque meningiomas and meningiomas related to other tumors as in neurofibromatosis.

\section{MRI imaging}

For cranial MRI examinations at dr. Moewardi Hospital, Surakarta, a 1.5 T MRI scanner (GEMR HDXT) is used, with a standard head coil. Pre-contrast T1-weighted spin-echo (SE) (TR/TE 401/8.5 ms), T2-weighted fast SE (FSE) (TR/TE 5256/73.8 ms), fluid-attenuated inversion recovery (FLAIR) (TR/TE/9000/145.2 $\mathrm{ms}$ ) and DWI sequences (TR/TE 5200/81.5 ms) are all taken in axial section. The matrix used is $256 \times 256$ with a field of view (FOV) of $25 \mathrm{~cm}$, slice thickness of $3 \mathrm{~mm}$, and a $3-\mathrm{mm}$ gap between slices. Contrast-enhanced T1-weighted images are obtained in axial section after the patient has been given IV contrast (Gadovist, 1 $\mathrm{mmol} / \mathrm{ml}$ ). The ADC folders are processed from DWI using Functool (GE Medical System) software with b-values of 0 and $1000 \mathrm{~s} /$ $\mathrm{mm}^{2}$. Simultaneously, for the ADC value calculation the RadiAnt DICOM Viewer (64bit) (Medixant Company, Poznan, Poland) is used. Data collection and analysis is done using Microsoft Office 2016 (Microsoft Corporation, Redmond, WA, USA) and IBM ${ }^{\circledR}$ SPSS statistics version 25 (Released 2017, Armonk, NY: IBM). In the calculation of ADC values, we use RadiANT DICOM Viewer software by creating regions of interest (ROIs) on the solid parts of the tumor, guided by contrast sequence to assess 
tumor boundaries and FLAIR sequence to assess the limit of peritumoral edema. The ADC value is expressed in units of $x 10^{-3} \mathrm{~mm}^{2} / \mathrm{s}$. The calcified parts of the tumor are avoided. The assessment is also conducted on the tumor-free contralateral side as control, called normal appearing white matter (NAWM).

\section{Histopathological analysis}

The pathology preparations of the patients were re-evaluated according to the WHO 2016 classification. The WHO categorizes the malignancy grade of meningiomas based on their histopathological features, consisting of WHO grade I (typical), WHO grade II (atypical), and WHO grade III (anaplastic). WHO grade I (typical) meningiomas occur in almost $90 \%$ of the population, WHO grade II (atypical) meningiomas have an incidence of $5 \%-7 \%$ of the population, and WHO grade III (anaplastic) meningiomas account for $1 \%-3 \%$ of the population. Each type has many subtypes, namely nine benign subtypes (WHO grade I), three intermediate subtypes (WHO grade II), and three malignant subtypes (WHO grade III). This division is based on histopathological appearance. ${ }^{(12)}$ For the more aggressive subtypes, it is unclear what percentage of the histology must be present before being classified as malignant. However, chordoid, clear cell, papillary and rhabdoid subtypes are declared to be categorized as WHO grade II and WHO grade III if the morphological picture is $>50 .^{(12)}$ The level of malignancy referred to in this study are the two groups of benign and malignant meningiomas. Benign meningiomas are WHO grade I (typical) meningiomas, while malignant meningiomas are WHO grade II (atypical) and III (anaplastic) WHO meningiomas.

\section{Statistical analysis}

Since the data distribution was normal, we used an independent t-test for the hypothesis. To test the reliability of the measurement results of 2 observers in this study to assess whether it has a good suitability/reliability or not, the researchers made Bland Altman graphs ${ }^{(13)}$ and calculated the average difference, $95 \% \mathrm{CI}$ and limit of agreement between observers. The receiver operating characteristic (ROC) curve was analysed to determine significant cut-off points in distinguishing benign and malignant meningiomas.

\section{Ethical clearance}

This study was approved by the Board of Health Research Ethics Committee of Dr. Moewardi General Hospital 321/II/HREC/2020.

\section{RESULTS}

The characteristics of the subjects were described in Table 1. The study specimens consisted of 11 malignant (34.4\%) and 21 benign (65.6\%). Of these 32 specimens, most were from patients over 30 years old (90.6\%), and 24 $(75 \%)$ were from women. There were no significant differences in age, sex, and peritumoral edema between benign and malignant meningiomas ( $p>0.05$ ). From the histopathological examination results, only four specimens could not be assigned a known meningioma subtype. In this study, the most common subtypes were meningothelial, clear cell, and fibroblastic meningiomas. In contrast, the least common were lipomatous, transitional, atypical, and rhabdoid meningiomas (data not shown).

The reliability/conformity test between the two observers was conducted by assessing the Bland-Altman scatter chart of average differences. From the observers' calculations, the average difference between the ADC assessments was -0.091 and the standard deviation 0.196. The differences between the ADC values from the two observers (diff) associated with their average ADC value (mmean), produce the Bland-Alman scatter diagram in Figure 1. There was only one observation that was outside the limit of agreement or outside the value of 1.96 times the standard deviation (dotted line), which means there is one observation (3.1\%) different from the other observations (outlier). Since only 3.1\% ( $\alpha<5 \%)$ observations 
Table 1. Comparison of subject characteristics and clinical features between benign and malignant meningioma $(\mathrm{n}=32)$

\begin{tabular}{lccc}
\hline & Benign $(\mathbf{n}=\mathbf{2 1}, \mathbf{\%})$ & Malignant $(\mathbf{n}=\mathbf{1 1}, \mathbf{\%})$ & p value* \\
\hline Age (years) & & & \\
$\quad<30$ & $0(0.0)$ & $3(100.0)$ & $0.282^{\mathrm{a}}$ \\
$\quad \geq 30$ & $21(72.4)$ & $8(27.6)$ & \\
Gender & $5(62.5)$ & $3(37.5)$ & $0.829^{\mathrm{b}}$ \\
$\quad$ Male & $16(66.7)$ & $8(33.3)$ & \\
$\quad$ Female & $15(65.2)$ & $8(34.8)$ & $0.938^{\mathrm{b}}$ \\
Peritumoral edema & $6(66.7)$ & $3(33.3)$ & \\
$\quad$ Large size & $0.990 \pm 0.105 \times 10^{-3} \mathrm{~mm}^{2} / \mathrm{s}$ & $0.877 \pm 0.167 \times 10^{-3} \mathrm{~mm}^{2} / \mathrm{s}$ & $0.025^{\mathrm{c}}$ \\
$\quad$ Minimum & & \\
ADC & &
\end{tabular}

Abbreviation: ADC: apparent diffusion coefficient; ${ }^{\mathrm{a}} \mathrm{p}$ value $=$ Fisher exact test; ${ }^{\mathrm{b}} \mathrm{p}$ value $=$ Chi-square-test; ${ }^{\mathrm{c}} \mathrm{p}$ value $=$ independent$\mathrm{t}$ test; *Significant at $\mathrm{p}<0.05$

are outside the limit of agreement, we can conclude that the observers have good suitability/ reliability.

This study's data is numerical and of normal distribution based on the Shapiro-Wilk test. In the independent $t$-test, there is a statistical difference in the average $\mathrm{ADC}$ value of benign and malignant meningiomas. The average ADC value in malignant meningiomas is $0.887 \pm 0.167 \times 10$ ${ }^{3} \mathrm{~mm}^{2} / \mathrm{s}$, while the average ADC value in benign meningiomas is $0.990 \pm 0.105 \times 10^{-3} \mathrm{~mm}^{2} / \mathrm{s}$. Thus, the average $\mathrm{ADC}$ value in malignant meningiomas is lower than in benign meningiomas, and there is a significant difference between the average $\mathrm{ADC}$ values in malignant and benign meningiomas $(\mathrm{p}<0.05)$ (Table 1).

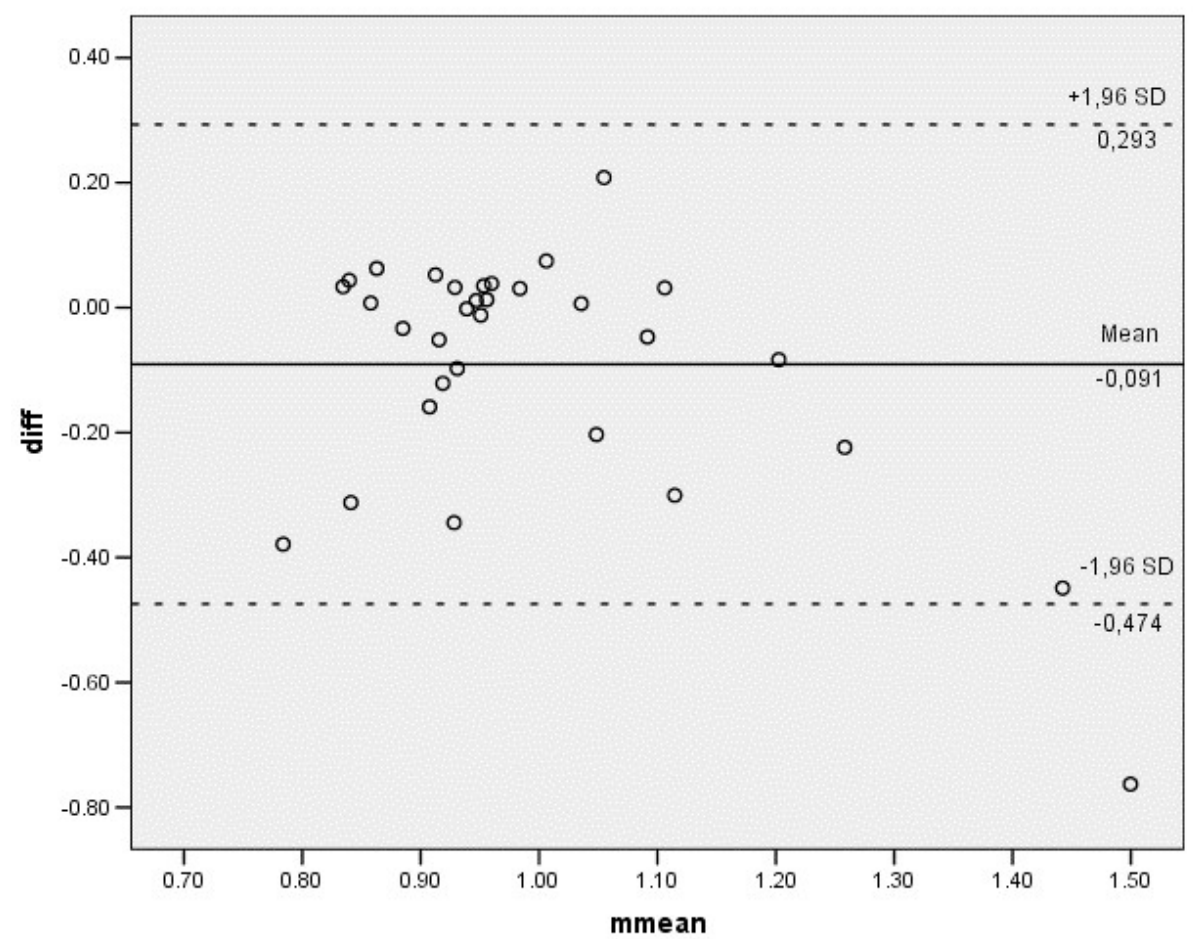

Figure 1. Bland-Altman scatter diagram. Diff is the difference between ADC values from two observers. Mmean is the average ADC value from the two observers. The dotted line is the limit of agreement or 1.96 times the standard deviation of the two observers. There is good suitability/reliability if the observation data from the two observers differ from the limit of agreement by less than $5 \%(\alpha<5 \%)$. 
ROC Curve

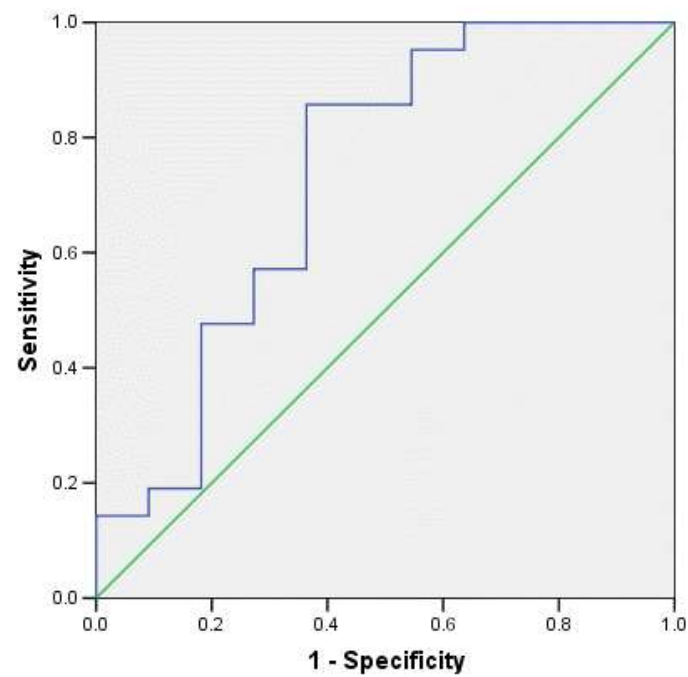

Figure 2. ROC curve of mean ADC value assessment. Larger ADC values indicate a benign lesion. The coordinates of the curves are the sensitivity and 1specificity of the variables. The variable is the ADC value taken by an observer
Since the average ADC value in benign and malignant meningiomas is significantly different from the benign meningiomas ADC value, which was higher than in malignant meningiomas, we look for the ROC curve's cutoff point, illustrated in Figure 2. From the ROC curve, the AUC value is $0.732(\mathrm{p}<0.05)$, with the cutoff point for ADC being $0.886 \times 10^{-3} \mathrm{~mm}^{2} / \mathrm{s}$ with a sensitivity of 0.857 and specificity of 0.636 . This can be interpreted to indicate that the strength of the ADC's diagnostic value in determining the malignancy grade of meningiomas is moderate, namely $73.20 \%(>70 \%-80 \%)$. Based on the sensitivity value, it can be interpreted that $85.70 \%$ of benign meningiomas can be indicated based on the ADC value of $>0.886 \times 10^{-3} \mathrm{~mm}^{2} / \mathrm{s}$. In contrast, the specificity value means that $63.6 \%$ of malignant meningiomas can be indicated by an $\mathrm{ADC}$ value of $<0.886 \times 10^{-3} \mathrm{~mm}^{2} / \mathrm{s}$.
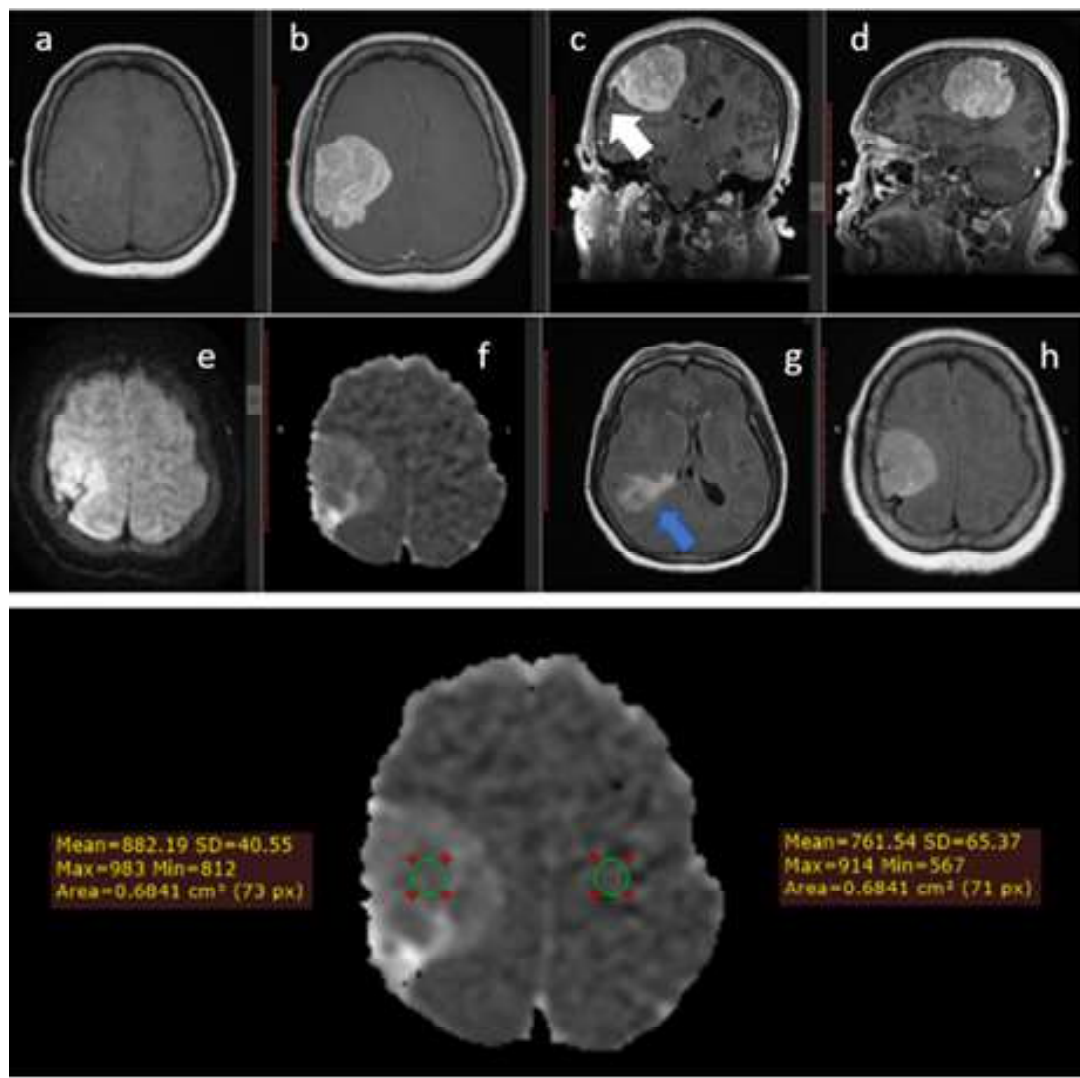

Figure 3. Female, 41 years old, complained about headache and weakness of the left side of the body

On MRI examination, the lesion was hypointense on T1WI (a), which on contrast (b, c, and d) showed contrast enhancement with a dural tail appearance (white arrow) in the convexity of the right parietal lobe. Peritumoral edema (blue arrow) was in the right temporal lobe on the T2 FLAIR sequence ( $\mathrm{g}$ and $\mathrm{h}$ ). The DWI examination showed fluid restriction (e), and a hypointense lesion appearing on the ADC (f) 

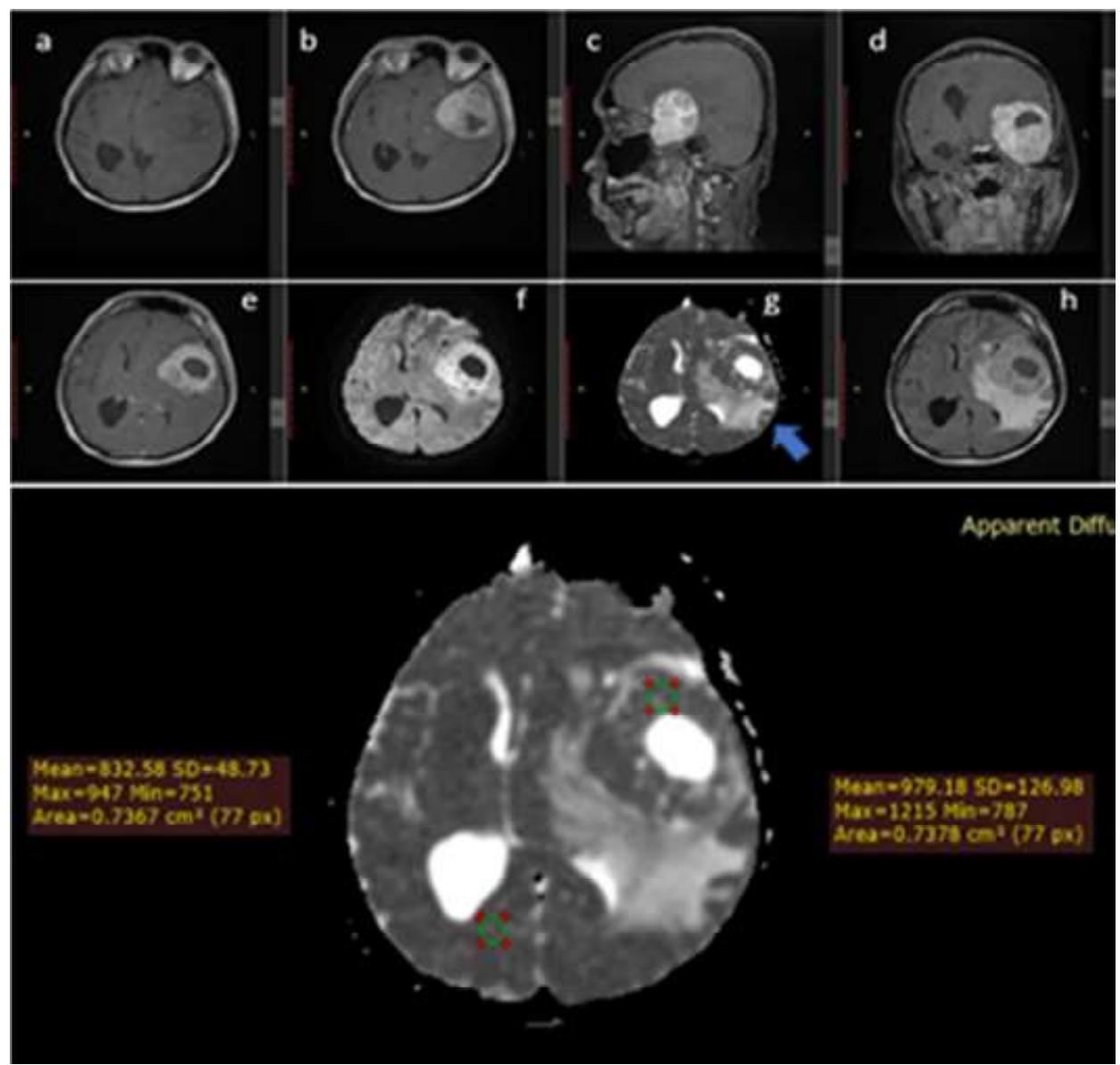

Figure 4. Male 34 years old, with chief complaints of seizures and visual disturbances. The MRI examination showed a heterointense lesion with a solid-cystic component on T1WI (a) and T1 FLAIR (h). When administered contrast (b, c, d, e), it showed contrast enhancement in the left frontotemporoparietal lobe. Peritumoral edema (blue arrow) is seen in the left frontotemporoparietal lobe. On DWI examination (f), there was a fluid restriction in the solid area (orange arrow) and a hypointense lesion in the solid area on the ADC (g)

The cutoff points of ADC value based on the ROC curve are divided into 2 categories for diagnostic test purposes: $>0.886 \times 10^{-3} \mathrm{~mm}^{2} / \mathrm{s}$ and $<0.886 \times 10^{-3} \mathrm{~mm}^{2} / \mathrm{s}$. The sensitivity of ADC value $>0.886 \times 10^{-3} \mathrm{~mm}^{2} / \mathrm{s}$ as the risk of malignant meningioma incidence is $63.6 \%$, and the specificity value is $85.7 \%$. In contrast, the PPV and NPV are $70 \%$ and $81.8 \%$, respectively.

One of our subjects was a 41 -year-old female, who complained of headache and weakness of the left side of the body. There was a tumor in the convexity of the right parietal lobe with peritumoral edema spreading through the right temporal lobe on MRI examination. The DWI examination showed fluid restriction, the ADC value was $0.882 \times 10^{-3} \mathrm{~mm}^{2} / \mathrm{s}$, and the NADC value was $0.762 \times 10^{-3} \mathrm{~mm}^{2} / \mathrm{s}$. If categorized based on the cutoff point this tumor leads to the diagnosis of a malignant meningioma $\left(<0.886 \times 10^{-3} \mathrm{~mm}^{2} / \mathrm{s}\right)$. It is consistent with her histopathological result of chordoid meningioma of WHO grade II (Figure 3).

Another subject was a 34-year-old male, with chief complaints of seizures and visual disturbances. The MRI examination showed a solid cystic tumor in the left fronto-temporoparietal lobe. The ADC value was found to be $0.979 \times 10-3 \mathrm{~mm}^{2} / \mathrm{s}$; if categorized based on our cutoff point, the diagnosis leads to a benign meningioma ( $\left.>0.886 \times 10-3 \mathrm{~mm}^{2} / \mathrm{s}\right)$ that is consistent with the histopathological results of angiomatous meningioma (WHO grade I) (Figure 4). 


\section{DISCUSSION}

In this study, the prevalence of malignant meningioma was higher than that of benign meningioma and this result was in line with a previous study conducted by Surov et al., ${ }^{(8)}$ which showed that benign meningioma has a higher incidence than malignant meningioma. Similar to the previous study of Sohu et al., ${ }^{(1)}$ there were more women among the subjects of the present study.

This study also showed a significant difference between ADC value in benign meningioma and malignant meningioma with the cutoff point at $0.886 \times 10^{-3} \mathrm{~mm}^{2} / \mathrm{s}$, which is close to the ADC cutoff point of $0.85 \times 10^{13} \mathrm{~mm}^{2} / \mathrm{s}$ in the studies of Sohu et al. ${ }^{(1)}$ and Surov et al..$^{(8)}$ The ADC measured using diffusion-weighted imaging sequence has been studied previously in predicting the histological nature of brain tumors. ${ }^{(14,15)}$ The ADC, which is calculated automatically by software, shows the magnitude of the diffusion of the water molecules. In tumors with a high proliferation of tumor cells, the diffusivity of water molecules is reduced. Therefore, an inverse correlation has been reported between ADC value and tumor cellularity due to this effect. ${ }^{(16)}$

The sensitivity in this study was $63.6 \%$, which was lower than that in the previous studies conducted by Abdel-Kerim et al. ${ }^{(17)}$ and Neethu et al., ${ }^{(18)}$ namely $81.2 \%$ and $84.0 \%$, respectively. A low sensitivity can be due to the number of benign and malignant subjects, as in the study by Abdel-Kerim et al. ${ }^{(17)}$ with 36 benign and 11 malignant samples, and in the study by Neethu et al. ${ }^{(18)}$ with 53 benign and 13 malignant samples. Another source of low sensitivity is the high average malignant ADC value close to the cutoff point, because the area of necrosis in malignant meningioma causes increasing fluid diffusion. For example, in one of the samples, where the ADC value is $1.067 \times 10^{-3} \mathrm{~mm}^{2} / \mathrm{s}$ when categorized by cutoff point, the diagnosis leads to a picture of benign meningioma $\left(>0.886 \times 10^{-3} \mathrm{~mm}^{2} / \mathrm{s}\right)$. However, histopathology results show a picture of clear cell meningioma with WHO grade II.
This study also obtained a specificity value of $85.7 \%$, greater than in the previous study of Surov et al., ${ }^{(8)}$ but smaller than in the study by AbdelKerim et al. ${ }^{(17)}$ with specificity of $91.7 \%$. The large sensitivity of the study of Abdel-Kerim ${ }^{(17)}$ may have been influenced by the fairly large average distance of benign ADC value from the cutoff point value of the benign meningioma average ADC value of $1.02 \pm 0.16 \times 10^{-3} \mathrm{~mm}^{2} / \mathrm{s}$ with a cutoff point value of $0.8 \times 10^{-3} \mathrm{~mm}^{2} / \mathrm{s}$. While in the research conducted by Surov et al., ${ }^{\left({ }^{(8)}\right.}$ the average $\mathrm{ADC}$ value in benign meningioma was $0.88 \pm 0.08 \times 10^{-3} \mathrm{~mm}^{2} / \mathrm{s}$ with the cutoff point at $0.85 \times 10^{-3} \mathrm{~mm}^{2} / \mathrm{s}$. In our study, the ADC value of benign meningioma was higher than that of malignant meningioma. These results were in agreement with those of the study of Hirunpat et al., ${ }^{(19)}$ where the average $\mathrm{ADC}$ value in the benign subgroup $\left(0.83 \times 10^{-3} \mathrm{~mm}^{2} / \mathrm{s} \pm 0.37 \times 10^{-3} \mathrm{~mm}^{2} /\right.$ s) was higher than in the malignant subgroup $\left(0.698 \times 10^{-3} \mathrm{~mm}^{2} / \mathrm{s} \pm 0.06 \times 10^{-3} \mathrm{~mm}^{2} / \mathrm{s}\right)$.

In the present study, PPV and NPV were $70.0 \%$ and $81.8 \%$, respectively, and not much different from those of previous studies, with PPV $85.7 \%$ and NPV 95\%. Similarly, in the study by Abdel-Kerim et al., ${ }^{(17)}$ PPV was $75 \%$ and NPV 94.3\%. Our low NPV compared to previous studies can be attributed to different study populations and necrotic malignancy images being mistaken for cystic components in the DWI picture of Sohu et al. ${ }^{(1)}$

Two of the present study's limitations are inadequately archived data and the observational nature of this study, while the population subtype variants are quite diverse. Another limitation is that we did not investigate the diagnostic accuracy of first-order histogram $(\mathrm{FOH})$ extracted features, which show pixels in an image with similar intensity at a defined intensity level. ${ }^{(20)}$

This study shows that the cutoff value of ADC from meningioma may help the clinician predict the diagnosis, but making a prompt diagnosis of meningioma is still based on the histopathological findings. However, the cutoff point obtained in this study may be expected to 
be useful as raw data for further research. In the radiology field, it could be a guide to be used in the daily protocol in MRI examination.

\section{CONCLUSIONS}

This study demonstrated that the ADC is a valid diagnostic tool. Further, mean ADC can be used as a good test to differentiate benign and malignant meningiomas and help the clinician predict pathological abnormalities due to the malignancy for evaluation before therapy.

\section{CONFLICT OF INTEREST}

The authors declare that they have no conflicts of interest.

\section{ACKNOWLEDGEMENTS}

The authors express their gratitude to Dr. Moewardi General Hospital's management and staff, Surakarta, especially the radiology department, for permission to analyze the samples in their MRI workstation.

\section{CONTRIBUTORS}

RRM conceived, designed, and did data collection, statistical analysis, and manuscript writing. RFR, HW, SS, and PSP designed, did the editing, reviewed, and did final approval of the manuscript. WS contributed to revision of the manuscript. All authors have read and approved the final the manuscript.

\section{REFERENCES}

1. Sohu DM, Sohail S, Shaikh R. Diagnostic accuracy of diffusion weighted MRI in differentiating benign and malignant meningiomas. Pak J Med Sci 2019; 35:726-30. doi: 10.12669/pjms.35.3.1011.

2. Lu Y, Liu L, Luan S, Xiong J, Geng D, Yin B. The diagnostic value of texture analysis in predicting WHO grades of meningiomas based on ADC maps: an attempt using decision tree and decision forest. Eur Radiol 2019;29:1318-28. doi: 10.1007/ s00330-018-5632-7.
3. Surov A, Ginat DT, Sanverdi E, et al. Use of diffusion weighted imaging in differentiating between malignant and benign meningiomas: a multicenter analysis. World Neurosurg 2016;88: 598-602. doi: 10.1016/j.wneu.2015.10.049.

4. Saligheh Rad H, Safari M, Kazerooni AF, Moharamzad Y, Taheri MS. Apparent diffusion coefficient (ADC) and first-order histogram statistics in differentiating malignant versus benign meningioma in adults. Iran J Radiol 2019; 16:e74324. doi: 10.5812/iranjradiol.7432416.

5. Baskan O, Silav G, Bolukbasi FH, Canoz O, Geyik S, Elmaci I. Relation of apparent diffusion coefficient with $\mathrm{Ki}-67$ proliferation index in meningiomas. Br J Radiol 2016;89:4-9. doi: 10.1259/ bjr.20140842.

6. Madhok R, Sachdeva P. Measurement of mean ADC values in tubercular vertebrae and associated collection. J Clin Diagn Res 2016;10: TC19-TC23. doi: 10.7860/JCDR/2016/20520.8344.

7. Moraru L, Dimitrievici L. Apparent diffusion coefficient of the normal human brain for various experimental conditions AIP Conference Proceedings 1796,2017.040005-1-040005-6. doi: 10.1063/1.4972383.

8. Surov A, Gottschling S, Mawrin C, et al. Diffusionweighted imaging in meningioma: Prediction of tumor grade and association with histopathological parameters. Transl Oncol 2015; 517-23. doi: 10.1016/j.tranon.2015.11.012.

9. Azeemuddin M, Nizamani WM, Tariq MU, Wasay M. Role of ADC values and ratios of MRI scan in differentiating typical from atypical/anaplastic meningiomas. J Pak Med Assoc 2018;68:1403-6.

10. Yiping L, Kawai S, Jianbo W, Li L, Daoying G, Bo Y. Evaluation parameters between intra-voxel incoherent motion and diffusion-weighted imaging in grading and differentiating histological subtypes of meningioma: a prospective pilot study. J Neurol Sci 2017;372:60-9. doi: 10.1016/ j.jns.2016.11.037.

11. Memon MA, Ting H, Cheah JH, Thurasamy R, Chuah F, Cham TH. Sample size for survey research: review and recommendations, Appl Struct Equation Modeling 2020;2920;4: i-xx11.

12. Vaubel RA, Chen SG, Raleigh DR, et al. Meningiomas with rhabdoid features lacking other histologic features of malignancy: a study of 44 cases and review of the literature. J Neuropathol Exp Neurol 2016;75:44-52. doi: 10.1093/jnen/nlv006.

13. Giavarina D. Understanding Bland Altman analysis. Biochem Med (Zagreb) 2015;25:141-51. doi: 10.11613/BM.2015.015.

14. Server A, Kulle B, Maehlen J, et al. Quantitative apparent diffusion coefficients in the 
characterization of brain tumors and associated peritumoral edema. Acta Radiol 2009;50:682-9. doi: 10.1080/02841850902933123.

15. Yin B, Liu L, Zhang BY, Li YX, Li Y, Geng DY. Correlating apparent diffusion coefficients with histopathologic findings on meningiomas. Eur J Radiol 2012;81:4050-6. doi: 10.1016/j.ejrad.2012. 06.002 .

16. Chen L, Liu M, Bao J, et al. The correlation between apparent diffusion coefficient and tumor cellularity in patients: a meta-analysis. PLoS One 2013;8. e79008. doi: 10.1371/journal.pone.0079008.

17. Abdel-Kerim A, Shehata M, El Sabaa B, Fadel S, Heikal A, Mazloum Y. Differentiation between benign and atypical cranial meningiomas. Can ADC measurement help? MRI findings with hystopathological correlation. Egyptian J Radiol Nuclear Med 2018;49:172-5. doi: 10.1016/ j.ejrnm.2017.10.004.
18. Neethu PM, Saanida MP, Subramaniam G, et al. Use of MRI apparent diffusion coefficient to noninvasively differentiate between the histologic grades of meningioma. J Evolution Med Dent Sci 2020;9:1679-83. doi: 10.14260/jemds/2020/ 369.

19. Hirunpat S, Sanghan N, Watcharakul C, Kayasut $\mathrm{K}$, Ina N, Pornrujee H. Is apparent diffusion coefficient value measured on picture archiving and communication system workstation helpful in prediction of high-grade meningioma? Hong Kong J Radiol 2016;19:84-90. doi: 10.12809/ hkjr1615346.

20. Just N. Improving tumour heterogeneity MRI assessment with histograms. Br J Cancer 2014; 111:2205-13. doi: 10.1038/bjc.2014.512. 